\title{
GROWTH OF SOLUTIONS OF LINEAR DIFFERENTIAL EQUATIONS AT A LOGARITHMIC SINGULARITY
}

BY

A. ADOLPHSON, B. DWORK AND S. SPERBER

\begin{abstract}
We consider differential equations $Y^{\prime}=A Y$ with a regular singular point at the origin, where $A$ is an $n \times n$ matrix whose entries are $p$-adic meromorphic functions. If the solution matrix at the origin is of the form $Y=P \exp (\theta \log x)$, where $P$ is an $n \times n$ matrix of meromorphic functions and $\theta$ is an $n \times n$ constant matrix whose Jordan normal form consists of a single block, then we prove that the entries of $P$ have logarithmic growth of order $n-1$.
\end{abstract}

Let $\Omega$ be an algebraically closed field of characteristic zero complete under a nonarchimedean valuation with residue classfield of characteristic $p$. Let $\Lambda$ be the ring of functions $u=f / g$ meromorphic on the open disk $D\left(0,1^{-}\right)$where $f$ and $g$ both converge on $D\left(0,1^{-}\right)$but $g$ is bounded on the disk. Let $\Lambda_{0}$ be a subring of $\Lambda$ which satisfies conditions (3.1)-(3.5) below. The standard example would be the ring $\Lambda(K)$ of functions defined over a discrete valuation subfield $K$ of $\Omega$ which lie in $\Lambda$. In this standard example the elements of $\Lambda(K)$ have only a finite number of poles in $D\left(0,1^{-}\right)$.

Let

$$
d y / d x=A y
$$

be a system of linear differential equations where $A$ is an $n \times n$ matrix with coefficients in $\Lambda_{0}$. Suppose that the origin is a regular singular point and that the solution matrix at the origin is of the form

$$
Y=P \exp (\theta \log x)
$$

where $P$ is an $n \times n$ matrix with entries in $\Lambda_{0}$ and $\theta$ is an $n \times n$ constant matrix. Suppose furthermore that the determinant of $P$ is bounded as an element of $\Lambda_{0}$. Generalizing a conjecture of Dwork [3], we ask whether the entries of $P$ have logarithmic growth of order $n-1$ (for definitions see $\S 1$ ). The present work provides an affirmative answer provided the matrix $\theta$ has Jordan normal form consisting of a single block, i.e. $\log ^{n-1} x$ appears in the formal solution of $(0.1)$.

This problem can be resolved in cases arising from geometry along the lines outlined in [2, Theorem 6]. In the present work we make no hypothesis concerning the existence of Frobenius structure. 
This question is related to the work of Dwork-Robba [4] which will be used here. Their work gave information only in disks free of singularities and so cannot be applied directly.

We thank E. Bombieri for stimulating renewed interest in this question.

\section{Definitions.}

1.1. $\Omega$ is an algebraically closed field of characteristic zero complete under a nonarchimedean valuation with residue classfield of characteristic $p$.

1.2. For each $a \in \Omega$ and each positive real number $r$, let $D\left(a, r^{-}\right)=\{x \in \Omega \mid$ $|x-a|<r\}$. For $f \in \Omega[[x-a]], f=\sum_{\nu=0}^{\infty} b_{\nu}(x-a)^{\nu}$, analytic on $D\left(a, r^{-}\right)$, we define for $0 \leqslant \rho<r$,

$$
|f|_{a}(\rho)=\operatorname{Sup}_{\nu}\left|b_{\nu}\right| \rho^{\nu}=\sup _{|x-a|=\rho}|f(x)| .
$$

This is extended to functions $u=f / g$ meromorphic on $D\left(a, r^{-}\right)$by writing $|u|_{a}(\rho)$ $=|f|_{a}(\rho) /|g|_{a}(\rho)$.

1.3. $\mathfrak{A}_{0}$ is the ring of elements of $\Omega[[x]]$ which converge on $D\left(0,1^{-}\right)$.

$\mathfrak{A}_{0}^{\prime}$ is the field of quotients of $\mathfrak{A}_{0}$.

$\mathfrak{B}_{0}$ is the ring of all $u \in \mathfrak{U}_{0}$ which are bounded as functions on $D\left(0,1^{-}\right)$.

$\Lambda$ is the ring of all $u \in \mathfrak{A}_{0}^{\prime}$ of the form $u=f / g$ where $f \in \mathfrak{A}_{0}, g \in \mathfrak{B}_{0}$.

1.4. We say that $u \in \Lambda$ has logarithmic growth $\alpha(\geqslant 0)$ if

$$
|u|_{0}(r)=O\left(\left(\log \frac{1}{r}\right)^{-\alpha}\right) \quad \text { as } r \rightarrow 1^{-} \text {. }
$$

An element $u=f / g$ of $\Lambda$ is said to be bounded if $f, g$ lie in $\mathfrak{B}_{0}$. This is the same as saying that $u$ is of zero logarithmic growth.

2. Generalities for scalar equations with a logarithmic singularity. Let $L$ be an $n$th order ordinary linear differential operator with coefficients in $\Lambda_{0}$ and with regular singularity at the origin. We assume the solution space at the origin is spanned by $n$ functions $\left\{y_{1}, \ldots, y_{n}\right\}$ having the following form:

$$
Y=\left(y_{1}, \ldots, y_{n}\right)=\left(f_{1}, \ldots, f_{n}\right) \exp (H \log x)
$$

where each $f_{i} \in \Lambda_{0}$ and $H$ is the constant matrix $H_{i, j}=\delta_{i, j-1}$.

LEMMA.

(2.1) There exists a sequence $\left\{L_{i}\right\}_{i=0}^{n-1}$ of elements of $\mathfrak{U}_{0}^{\prime}[D]$ such that

$$
L_{0}=L, \quad \operatorname{order} L_{i}=n-i \text {. }
$$

(2.1.2) A basis of the solution space of $L_{i}$ at the origin is given by the $n-i$ functions $\left\{y_{i, 1}, \ldots, y_{i, n-i}\right\}$ having the following form

$$
Y_{i}=\left(y_{i, 1}, \ldots, y_{i, n-i}\right)=F_{i} \exp H^{(i)} \log x
$$

where $F_{i}=\left(f_{i, 1}, \ldots, f_{i, n-i}\right) \in \mathfrak{U}_{0}^{\prime n-i}$ and $H^{(i)}$ is the $(n-i) \times(n-i)$ matrix

$$
\begin{gathered}
H_{j, k}^{(i)}=\delta_{j, k-1}, \quad 1 \leqslant j, k \leqslant n-i, \\
y_{t, 1}^{-1} \circ L_{t} \circ y_{t, 1}=L_{t+1} \circ D \quad(t=0,1, \ldots, n-2) .
\end{gathered}
$$


(2.2) For $k=0,1, \ldots, n-1$ we construct a Frobenius decomposition $[5, p .120]$ of $L_{k}$ by setting

$$
\begin{aligned}
y_{k, 1} & =v_{k, 1}, \\
y_{k, 2} & =v_{k, 1} D^{-1} v_{k, 2}, \\
& \cdots, \\
y_{k, n-k} & =v_{k, 1} D^{-1} v_{k, 2} \cdots D^{-1} v_{k, n-k} .
\end{aligned}
$$

Then

$$
\begin{gathered}
v_{0, j}=v_{k, j-k}, \quad 0 \leqslant k \leqslant n-1, k \leqslant j \leqslant n . \\
v_{k, j} \in \mathfrak{A}_{0}^{\prime}, \quad 0 \leqslant k \leqslant n-1,1 \leqslant j \leqslant n-k . \\
v_{0, j+2} \text { has residue } 1 \text { at } x=0 \quad(0 \leqslant j \leqslant n-2) .
\end{gathered}
$$

(2.3) Let $w_{k}$ be the wronskian of $Y_{k}=\left(y_{k, 1}, \ldots, y_{k, n-k}\right)$. Then $w_{k}=w_{k+1} y_{k, 1}^{n-k}$.

(2.4) The wronskian $\tilde{w}$ of $\left\{y_{1}, \ldots, y_{n-1}\right\}$, the first $n-1$ functions of $Y_{0}$, lies in $\Lambda_{0}$. The wronskian $w=w_{0}$ of $Y_{0}$ lies in $\Lambda_{0}$.

Proof. We set $L_{0}=L, Y_{0}=Y, F_{0}=F$ and observe that property (2.1.2) holds for $i=0$.

We now use induction and suppose $L_{i}, Y_{i}, F_{i}$ defined for $0 \leqslant i \leqslant t$ and that (2.1.2) is valid in this range.

We define $L_{t+1}$ by property (2.1.3), the factorization on the right side being justified since the left side annihilates constants. It follows from (2.1.3) that a basis $Y_{t+1}=\left(y_{t+1,1}, \ldots, y_{t+1, n-t-1}\right)$ of solutions of $L_{t+1}$ is given by

$$
y_{t+1, j}=D\left(y_{t, j+1} / y_{t, 1}\right), \quad 1 \leqslant j \leqslant n-t-1 .
$$

We know that

$$
\left(1 / y_{t, 1}\right) Y_{t}=\left(1 / f_{t, 1}\right) F_{t} \exp \left(H^{(t)} \log x\right)
$$

and hence by (2.5)

$$
\left(0, Y_{t+1}\right)=\left(D\left(f_{t, 1}^{-1} F_{t}\right)+x^{-1} f_{t, 1}^{-1} F_{t} H^{(t)}\right) \exp \left(H^{(t)} \log x\right) .
$$

We now define

$$
f_{t+1, k}=D\left(f_{t, 1}^{-1} f_{t, k+1}\right)+x^{-1} f_{t, 1}^{-1} f_{t, k}, \quad 1 \leqslant k \leqslant n-t-1
$$

and set $F_{t+1}=\left(f_{t+1,1}, \ldots, f_{t+1, n-t-1}\right)$ and conclude that (2.1.3) is verified for $i=t+$ 1. This concludes the verification of (2.1).

To prove (2.2.1) we show

$$
v_{k, j}=v_{k+1, j-1}, \quad 0 \leqslant k<n-2, \quad 2 \leqslant j \leqslant n-k .
$$

By definition (2.2),

$$
\begin{gathered}
y_{k, j}=v_{k, 1} D^{-1} v_{k, 2} \cdots D^{-1} v_{k, j}, \\
y_{k+1, j-1}=v_{k+1,1} D^{-1} v_{k+1,2} \cdots D^{-1} v_{k+1, j-1} .
\end{gathered}
$$

By (2.5),

$$
y_{k+1, j-1}=D\left(y_{k, j} / y_{k, 1}\right),
$$


and by definition, $y_{k, 1}=v_{k, 1}$. Substituting (2.6) in (2.8) gives

$$
y_{k+1, j-1}=v_{k, 2} D^{-1} v_{k, 3} \cdots D^{-1} v_{k, j} \text {. }
$$

Comparing (2.7) with (2.9) for $j=2$ demonstrates (2.2.1.1) for $j=2$ and the assertion follows by induction on $j$ by means of the same comparison. This completes the verification of (2.2.1). To verify (2.2.2) we use (2.2.1) to write

$$
v_{k, j}=v_{k+j-1,1}=y_{k+j-1,1}=f_{k+j-1,1}
$$

which completes the proof of (2.2.2).

Assertion (2.2.3) is central for our application. By (2.2.1) and the definitions, for $0 \leqslant j \leqslant n-2$ we have

$$
\begin{aligned}
y_{j, 1} & =f_{j, 1}, \quad y_{j, 2}=f_{j, 1} \log x+f_{j, 2}, \\
v_{0, j+2} & =v_{j, 2}=D\left(y_{j, 2} / v_{j, 1}\right)=D\left(y_{j, 2} / y_{j, 1}\right),
\end{aligned}
$$

and hence

$$
v_{0, j+2}=\frac{1}{x}+D\left(f_{j, 2} / f_{j, 1}\right) .
$$

This completes the proof of (2.2.3).

To verify $(2.3)$ we note that by a classical formula $[\mathbf{5}$, p. $120 ; \mathbf{4}, \S 3.10 .1]$

$$
w_{k}=v_{k, 1}^{n-k} v_{k, 2}^{n-k-1} \cdots v_{k, n-k} .
$$

Relation (2.3) now follows by application of (2.2.1) and use of (2.11) for $w_{k}$ and $w_{k+1}$.

To verify (2.4) we observe that by truncation of (2.0)

$$
\left(y_{1}, \ldots, y_{n-1}\right)=\left(f_{1}, \ldots, f_{n-1}\right) \exp (\tilde{H} \log x),
$$

$\tilde{H}$ being obtained from $H$ by discarding the last row and the last column. Letting $\tilde{E}$ denote the operation

$$
\left(f_{1}, \ldots, f_{n-1}\right) \rightarrow D\left(f_{1}, \ldots, f_{n-1}\right)+x^{-1}\left(f_{1}, \ldots, f_{n-1}\right) \tilde{H},
$$

we compute the wronskian matrix of $\left(y_{1}, \ldots, y_{n-1}\right)$ to be

$$
\left(\begin{array}{c}
\tilde{F} \\
\tilde{E} \tilde{F} \\
\vdots \\
\tilde{E}^{n-1} \tilde{F}
\end{array}\right) \exp (\tilde{H} \log x) .
$$

The assertion is now clear since the entries of $\tilde{F}$ lie in $\Lambda_{0}$. A similar argument shows that $w\left(=w_{0}\right)$ lies in $\Lambda_{0}$.

3. Generalities for meromorphic functions. See $\S 1.3$ for the definition of $\Lambda$.

Lemma. (3.1) If $\xi$ and $\eta$ lie in $\Lambda, \eta$ bounded, then $\xi / \eta$ lies in $\Lambda$.

(3.2) If $\xi$ and $\eta$ lie in $\Lambda$ and if $\xi \eta$ is bounded, then $\xi$ and $\eta$ are each bounded.

(3.3) If $\xi \in \mathfrak{A}_{0}^{\prime}, D \xi \in \Lambda$, then $\xi \in \Lambda$.

(3.4) If $\xi \in \Lambda$, $\xi$ has $\log$ growth $\alpha$, then $D \xi \in \Lambda$ and has $\log$ growth $\alpha$.

We do not know whether $\Lambda$ satisfies the following condition. 
(3.5) If $\xi \in \Lambda, D \xi$ has logarithmic growth $\alpha$, then $\xi$ has logarithmic growth $\alpha+1$.

Proof. If $\xi$ and $\eta$ lie in $\Lambda$ then $\xi=f_{1} / g_{1}, \eta=f_{2} / g_{2}$, with $g_{1}$ and $g_{2}$ in $\mathfrak{B}_{0}$. If $\eta$ is bounded then $f_{2} \in \mathfrak{B}_{0}$ and so $\xi / \eta=f_{1} g_{2} / g_{1} f_{2} \in \Lambda$. If with the same notation, we do not know that $\eta$ is bounded but do know that $\xi \eta$ is bounded, then we conclude that $f_{1} f_{2}$ is bounded as an analytic function on $D\left(0,1^{-}\right)$. As $\left|f_{i}\right|_{0}(r)$ increases monotonically with $r$ as $r \rightarrow 1^{-}$, and since $\left|f_{1} f_{2}\right|_{0}(r)=\left|f_{1}\right|_{0}(r)\left|f_{2}\right|_{0}(r)$, it is clear that $\left|f_{i}\right|_{0}(r)$ is bounded as $r \rightarrow 1^{-}$.

To demonstrate (3.3), we write $\xi=u / v, D \xi=f / g, f, u, v \in \mathfrak{A}_{0}, g \in \mathfrak{B}_{0}$. For each $r \in(0,1)$ let $N_{v}(r)$ (resp, $N_{g}(r)$ ) denote the number of zeroes (counting multiplicity) of $v$ (resp, $g$ ) in $D\left(0, r^{-}\right)$. If $z$ is a zero of $v$ of order $s \geqslant 1$ then it is a pole of $D \xi$ of order $s+1$ and hence a zero of $g$ of that order. Thus $N_{g}(r) \geqslant N_{v}(r)$. We fix $\varepsilon \in(0,1)$ and compute for $t \in(\varepsilon, 1)$,

$$
\begin{aligned}
\log |v|_{0}(t) & /(v)_{0}(\varepsilon)=\int_{\varepsilon}^{t} N_{v}(r) d(\log r) \\
& \leqslant \int_{\varepsilon}^{t} N_{g}(r) d(\log r)=\log |g|_{0}(t) /|g|_{0}(\varepsilon)
\end{aligned}
$$

(we do not set $\varepsilon=0$ since 0 might be a zero of $v$ or of $g$ ). Since $g \in \mathfrak{B}_{0}$, we see that the same holds for $v$. This completes the proof.

Corollary. Let $K$ be a subfield of $\Omega$ with discrete valuation ring. Let $\Lambda(K)$ be the set of all elements $f / g \in \Lambda$ with $f$ and $g$ in $K[[x]], f \in \mathfrak{A}_{0}, g \in \mathfrak{B}_{0}$. Then $\Lambda(K)$ satisfies conditions (3.1)-(3.5).

Proof. It is enough to verify (3.5). It follows from the theory of the Newton polygon [1] that each $g \in \mathfrak{B}_{0} \cap K[[x]]$ is a product of a polynomial $g_{1}$ with an element $g_{2}$ of $\mathfrak{U}_{0}$ having no zero in $D\left(0,1^{-}\right)$. Hence each element $\xi$ of $\Lambda(K)$ is an analytic function on some annulus $\{x|a<| x \mid<1\}$. (This annulus will in general depend upon the element $\xi$.) The Laurent expansion $\xi=\sum_{s=-\infty}^{\infty} b_{s} x^{s}$ in this annulus has the property that for $r \in(a, 1)$, and as $r \rightarrow 1^{-}$,

$$
|\xi|_{0}(r)=O\left(\sup _{s \geqslant 0}\left|b_{s}\right| r^{s}\right) \text {. }
$$

If $D \xi$ has logarithmic growth $\alpha$ then

$$
\left|b_{s} s\right|=O\left(s^{\alpha}\right), \quad s>0,
$$

and hence $\left|b_{s}\right|=O\left(s^{\alpha+1}\right)$. This completes the proof.

4. $p$-adic estimates for a scalar equation with logarithmic singularity. We formulate our main result in terms of scalar equations.

THEOREM. Let $L$ be an ordinary nth order linear differential equation with coefficients in $\Lambda_{0}$ whose solution space at the origin is spanned by

$$
\left(y_{1}, \ldots, y_{n}\right)=\left(f_{1}, \ldots, f_{n}\right) \exp (H \log x)
$$

where $H$ is the $n \times n$ matrix $H_{i, j}=\delta_{i, j-1}$ and $f_{1}, \ldots, f_{n}$ are elements of $\Lambda_{0}$. We assume that the wronskian $w$ of $y_{1}, \ldots, y_{n}$ is a bounded element of $\Lambda_{0}$. The conclusion is that $f_{i}$ has logarithmic growth $i-1(1 \leqslant i \leqslant n)$. 
Proof. The equation $L$ satisfies the hypothesis of $\$ 2$. We will use the results and notation of that section without further explanation. The main point in the proof is to show that

4.1. $f_{i, 1}$ is a bounded element of $\Lambda_{0}(0 \leqslant i<n-1)$. We give two proofs of this assertion.

Proof I. Let $\{x|| x \mid=r(<1)\}$ define a set in $\Omega(r \neq 0)$ on which $v_{0, j}$ has no pole. Equation (2.10) shows that in the Laurent series representation of $v_{0, j}$ on this set, the term $1 / x$ appears if $j \geqslant 2$. This shows that

$$
\left|v_{0, j}\right|_{0}(r) \geqslant 1 / r \quad(j \geqslant 2) .
$$

Equation (2.11) with $k=0$ now shows that

$$
\left(\left|v_{0,1}\right|_{0}(r)\right)^{n} r^{-\left(\begin{array}{c}
n \\
2
\end{array}\right)} \leqslant|w|_{0}(r) .
$$

Letting $r \rightarrow 1^{-}$and recalling that $v_{0,1}=f_{1} \in \Lambda_{0}$, we obtain

$$
\left|v_{0,1}\right|_{0}(1) \leqslant\left(|w|_{0}(1)\right)^{1 / n}
$$

which shows that $f_{1}$ is a bounded element of $\Lambda_{0}$.

We now use (2.3) to deduce that $w_{1}=w_{0} / v_{0,1}^{n}$ is a ratio of bounded elements of $\Lambda_{0}$ and hence again a bounded element of $\Lambda_{0}$. We conclude from (2.5.1) and (3.1) and (3.4) (with $t=0$ ) that the entries of $F_{1}$ lie in $\Lambda_{0}$. We now conclude that $L_{1}$ satisfies the conditions of the present theorem and hence deduce relation 4.1 by induction on $n$.

ProOF II. Let $a \in D\left(0,1^{-}\right), a \neq 0$ be such that

(4.2.1) $L$ has no singularities in the disk $D\left(a,|a|^{-}\right)$,

(4.2.2) $f_{1}, \ldots, f_{n}$ have no poles in this disk.

Let $\tilde{w}$ be the wronskian of $\left(y_{1}, y_{2}, \ldots, y_{n-1}\right)$. Let $\log x / a$ denote the solution of the equation $d z / d x=1 / x$ analytic on the disk of (4.2.1) and taking the value 0 at $x=a$.

Let $u_{a}$ be the solution of $L$ at $x=a$ which satisfies the initial conditions:

$$
u_{a}^{(j)}(a)= \begin{cases}0, & 0 \leqslant j<n-1, \\ (n-1) !, & j=n-1 .\end{cases}
$$

Clearly $u_{a}$ is a linear combination with constant coefficients of the entries of $\left(f_{1}, \ldots, f_{n}\right) \exp (H \log x / a)$ and by an explicit calculation

$$
u_{a}(x)=f_{1}(x) \beta(a)\left(\log \frac{x}{a}\right)^{n-1}+H_{a}(x)
$$

with $\beta(a)=\tilde{w}(a) / w(a)$ and where $H_{a}$ is a linear combination with constant coefficients (depending upon $a$ ) of products of the form $f_{i}(\log x / a)^{\nu}$ with $\nu \leqslant n-2$. Since $w$ is bounded and by (2.4) $\tilde{w} \in \Lambda_{0}$ we know that $\beta \in \Lambda_{0}$.

For $s \geqslant 0$ we write

$$
\frac{1}{s !} D^{s} \equiv \sum_{j=0}^{n-1} G_{s, j} \frac{1}{j !} D^{j} \bmod L
$$

where the $G_{s, j}$ lie in the differential ring generated over $\mathbf{Q}$ by the coefficients of $L$. Thus the $G_{s, j}$ lie in $\Lambda_{0}$ and have poles only at the singularities of $L$. 
By Taylor's theorem and our choice of initial conditions for the solution $u_{a}$,

$$
u_{a}(x)=\sum_{s=0}^{\infty} G_{s, n-1}(a)(x-a)^{s} .
$$

The main point is that

$$
\left|H_{a}(x) /\left(\log \frac{x}{a}\right)^{n-1}\right|_{a}(r) \rightarrow 0
$$

as $r \rightarrow|a|^{-}$. On the other hand it follows from $[4,(5.1)]$ that

$$
\left|a^{s} G_{s, n-1}(a)\right| \leqslant C s^{n-1}
$$

where $C$ is a constant independent of $a$. This may be used together with (4.2.6) to show that

$$
\limsup _{r \rightarrow|a|^{-}}\left|u_{a}(x) /\left(\log \frac{x}{a}\right)^{n-1}\right|_{0}(r)<C^{\prime}
$$

with $C^{\prime}$ independent of $a$. Substituting (4.2.4) in this relation and applying (4.2.7) shows that

$$
\left|\beta(a) f_{1}(a)\right|<C^{\prime}
$$

for all $a$ satisfying (4.2.1), (4.2.2). This shows that $\beta$ and $f_{1}$ are elements of $\Lambda_{0}$ whose product is bounded and hence, by $\S 3, f_{1}$ is a bounded element of $\Lambda_{0}$. The second proof of (4.2) is now completed by induction precisely as in the first proof.

Proof of THEOREM. Assume that $f_{t, k}$ is an element of $\Lambda_{0}$ with log growth $k-1$ for fixed $k$ and $0 \leqslant t \leqslant n-1$. This has been verified for $k=1$. Equation (2.5.1) shows that $f_{t, k+1}$ may be computed from $f_{t+1, k}, f_{t, k}$, and $f_{t, 1}$ by integration. Thus in particular $f_{t, k} / x f_{t, 1}$ has by (3.1) $\log$ growth $k-1$ and lies in $\Lambda_{0}$. The same holds for $f_{t+1, k}$. On the other hand $f_{t, k+1} / f_{t, 1}$ lies in $\mathfrak{U}_{0}^{\prime}$. Hence by (2.5.1) and (3.3), $f_{t, k+1} / f_{t, 1}$ lies in $\Lambda_{0}$ and by (3.5) has $\log$ growth $k$. Hence $f_{t, k+1}$ lies in $\Lambda_{0}$ and has $\log$ growth $k$ for $0 \leqslant t \leqslant n-1$. This completes the proof of the theorem.

COROLLARY. Under the hypothesis of the theorem, suppose that at $x=0$ the vector $\left(f_{1}, \ldots, f_{n}\right)$ specializes to $\left(f_{1}, \ldots, f_{n}\right)(0)=(1,0, \ldots, 0)$. If we write

$$
f_{i}(x)=\sum_{j=0}^{\infty} a_{i, j} x^{j}
$$

then

$$
\left|a_{i, j}\right| \leqslant \sup _{1 \leqslant k \leqslant j} 1 /|k|^{i-1} .
$$

This is demonstrated by showing that each $f_{i, 1}$ in (2.1.2) is of the form

$$
f_{i, 1}=\dot{x}^{\lambda_{i}}\left(1+\sum_{s=1}^{\infty} c_{i, s} x^{s}\right)
$$

with $\lambda_{i} \in \mathbf{N},\left|c_{i, j}\right| \leqslant 1$. The corollary then follows from (2.5.1) by an inductive argument precisely as in the proof of the theorem. 
Note that the three functions $1, \log x, \operatorname{dilog}=\Sigma\left(x^{s} / s^{2}\right)$ satisfy a third order differential equation not covered by the hypothesis of this corollary but having growth of the type predicted by the conclusion of the corollary.

\section{REFERENCES}

1. Y. Amice, Les nombres p-adiques, Presses Universitaires de France, Paris, 1975.

2. B. Dwork, p-adic cycles, Inst. Hautes Etude Sci. Publ. Math. 37 (1969), 27-115.

3. __ On p-adic differential equations. II, Ann. of Math. (2) 98 (1973), 366-376.

4. B. Dwork and P. Robba, Effective p-adic bounds for solutions of homogeneous linear differential equations, Trans. Amer. Math. Soc. 259 (1980), 559-577.

5. E. L. Ince, Ordinary differential equations, Dover, New York, 1956.

Department of Mathematics, University of Washington, Seattle, Washington 98195

Department of Mathematics, Princeton University, Princeton, New Jersey 08544

Department of Mathematics, University of Minnesota, Minneapolis, Minnesota 55455 